\title{
Wenn Wahrheit wertlos wird
}

\section{Demonstrativer Bullshit in einer digitalisierten Gesellschaft}

\section{Romy Jaster und David Lanius}

„Zu den auffälligsten Merkmalen unserer Kultur gehört die Tatsache, daß es so viel Bullshit gibt.“ (Harry Frankfurt)

\section{$1 \quad$ Lügen und Bullshit in der Politik}

Politiker lügen von Zeit zu Zeit. 2002 äußerte Dick Cheney, der damalige Vizepräsident der USA, den berühmten Satz: „Simply stated, there is no doubt that Saddam Hussein now has weapons of mass destruction." Kurze Zeit später begannen die USA den zweiten Irakkrieg. Heute weiß man, dass es im Irak weder Massenvernichtungswaffen noch verlässliche Hinweise darauf gegeben hatte. Dick Cheney hat aller Wahrscheinlichkeit nach gelogen. Er hat die Öffentlichkeit wissentlich getäuscht, um ein politisches Ziel zu erreichen. Die angeblichen Massenvernichtungswaffen sollten den Einmarsch in den Irak legitimieren.

Einer weithin akzeptierten Definition der Lüge zufolge ist eine Lüge eine Behauptung, die der Sprecher für falsch hält und die mit der Absicht getätigt wird, den Adressaten in Bezug auf den Inhalt zu täuschen (Isenberg 1973, S. 248;

Der Beitrag stammt zu gleichen Teilen von beiden Autoren.

R. Jaster

Humboldt-Universität zu Berlin, Berlin, Deutschland

E-Mail: romy.jaster@hu-berlin.de

D. Lanius $(\varangle)$

Karlsruher Institut für Technologie (KIT), Karlsruhe, Deutschland

E-Mail: david.lanius@kit.edu 
Williams 2010, S. 98). ${ }^{1}$ Sie trifft auf Cheneys Äußerung wohl genauso zu wie auf viele Äußerungen im zeitgenössischen politischen Diskurs. So war Donald Trumps Behauptung, er habe nichts über die Zahlungen an die Pornodarstellerin Stormy Daniels durch seinen Anwalt Michael Cohen gewusst, aller Wahrscheinlichkeit nach eine Lüge. Nicht nur war die Behauptung tatsächlich falsch, man muss auch davon ausgehen, dass Trump mit seiner Behauptung eine Täuschungsabsicht verfolgte. Trump wusste über die Zahlung Bescheid, er wusste, dass Cohen sie getätigt hatte, um ihn und die Wahlkampagne vor negativen Schlagzeilen zu schützen, und er musste davon ausgehen, dass ihn seine Mitwisserschaft politisch in Bedrängnis bringen würde. Er selbst hielt die Behauptung also für falsch und stellte sie mit der Absicht auf, dass seine Adressaten sie für wahr hielten.

Neben klassischen Lügen offenbart Trumps öffentliche Kommunikation jedoch auch in besonderem Maße ein anderes Phänomen, das in der philosophischen Fachliteratur als „Bullshit“ bezeichnet wird. Harry Frankfurt (1986), der „Bullshit" als philosophischen Fachausdruck geprägt hat, unterscheidet Bullshitten von Lügen. Im Unterschied zum Lügner, der über die Tatsachen täuschen will, zeichnet sich Bullshit Frankfurt zufolge durch die vollständige Gleichgültigkeit des Sprechers gegenüber der Wahrheit aus.

Schauen wir uns Trumps Behauptung an, bei seiner Amtseinweihung habe es im Moment seiner Vereidigung zu regnen aufgehört und die Sonne sei herausgekommen. Tatsächlich regnete es durchgehend. In die gleiche Kategorie fällt seine Behauptung, die Menschenmenge bei besagter Amtseinweihung sei die größte gewesen, die es jemals bei der Amtseinweihung eines US-Präsidenten gegeben habe. An dieser Behauptung hielten er und sein Team auch dann noch fest, als längst eindeutige Fotoaufnahmen größerer Amtseinführungsfeiern vorlagen. Trumps Behauptung, er habe das beste Gedächtnis der Welt, scheint demselben Muster zu folgen.

In diesen Fällen ist es unplausibel zu unterstellen, Trump habe seine Zuhörerschaft hinsichtlich des behaupteten Sachverhalts täuschen wollen. Wir haben es hier also nicht mit Lügen zu tun. Vielmehr scheint es Trump schlichtweg gleichgültig zu sein, wie es sich in den genannten Fällen mit der Wahrheit verhält. Alle drei Fälle sind aller Wahrscheinlichkeit nach Bulllshit. Mit Blick auf Trumps

\footnotetext{
${ }^{1}$ Beide Bedingungen der genannten Definition von Lügen sind umstritten. Einige Autoren sind der Auffassung, dass es nicht genügt, dass der Lügner seine Behauptung für falsch hält. Vielmehr müsse die Behauptung auch tatsächlich falsch sein (siehe Carson 2006; Fallis 2009). Ebenfalls umstritten ist, ob Lügen tatsächlich immer mit einer Täuschungsabsicht einhergehen (siehe Carson 2006; Saul 2012).
} 
Behauptung, das beste Gedächtnis der Welt zu haben, spricht Frankfurt selbst sogar von geradezu ,possenhaft offensichtlichem Bullshit“ (Frankfurt 2016).

Trump mag der derzeit berühmteste politische Bullshitter sein, aber er ist offenkundig nicht der einzige. In diesem Beitrag werden wir uns ansehen, welche Funktion Bullshit gegenwärtig im politischen Diskurs spielt. Dazu werden wir zunächst genauer ausführen, was wir unter „Bullshit“" verstehen (Abschn. 2). Anschließend werden wir eine besondere Art von Bullshit herausstellen, die wir „demonstrativen Bullshit“ nennen (Abschn. 3). Wie wir argumentieren, kann diese Art von Bullshit von politischen Akteuren eingesetzt werden, um die Demokratie selbst zu unterminieren (Abschn. 4). Wir zeigen, dass die Digitalisierung den Handlungsspielraum dieser Akteure vergrößert (Abschn. 5), und skizzieren schließlich, wie die politische Bildung auf diese verhältnismäßig neue Situation reagieren könnte (Abschn. 6).

\section{Bullshit: Eine genauere Charakterisierung}

Wie genau verhalten sich nun Lügen und Bullshit zueinander? Das erschließt sich nicht unmittelbar und ist auch in der Literatur umstritten. ${ }^{2}$ In einer viel zitierten Passage schreibt Frankfurt dazu:

When an honest man speaks, he says only what he believes to be true; and for the liar, it is correspondingly indispensable that he considers his statements to be false. For the bullshitter, however, all these bets are off: he is neither on the side of the true nor on the side of the false. [...] He does not care whether the things he says describe reality correctly. He just picks them out, or makes them up, to suit his purpose. (Frankfurt 2005, S. 55)

Der Lügner orientiert sich also Frankfurt zufolge in dem Sinne an der Wahrheit, dass er zunächst einmal Überzeugungen über die Welt ausbilden muss, um in der Lage zu sein, zu lügen. Schließlich muss der Lügner glauben, dass seine Behauptung falsch ist, damit es sich überhaupt um eine Lüge handeln kann. Im Gegensatz dazu muss der Bullshitter keinerlei spezifische Überzeugungen über den behaupteten Sachverhalt haben. Um zu bullshitten, genügt es, Behauptungen aufzustellen, ohne die Tatsachen überhaupt in Betracht zu ziehen.

Was es genau heißt, dass der Bullshitter der Wahrheit gegenüber gleichgültig ist, lassen Frankfurts Ausführungen jedoch offen. Schließlich ist es auch dem

\footnotetext{
${ }^{2}$ Siehe Cohen (2002), Kimbrough (2006), Carson (2016), Wreen (2013) sowie Fallis und Stokke (2017).
} 
Bullshitter nicht unbedingt egal, $o b$ seine Behauptung wahr ist. Wir können sogar davon ausgehen, dass sich Bullshitter häufig wünschen, die Welt wäre ganz genau so, wie sie behaupten. Fallis und Stokke (2017) spezifizieren daher die charakteristische Gleichgültigkeit des Bullshitters gegenüber der Wahrheit auf hilfreiche Weise als eine Gleichgültigkeit gegenüber der Wahrheitsfindung mit Blick auf die zur Debatte stehende Fragestellung.

Stalnaker (2002) folgend können wir uns einen Diskurs als kooperatives Projekt vorstellen, dessen Ziel es ist, wahre Informationen zu sammeln. ${ }^{3}$ Vor diesem Hintergrund liegt Fallis und Stokkes Definition nahe. Der Definition nach zeichnet es den Bullshitter aus, dass er sich dem kooperativen Projekt der Wahrheitsfindung entzieht. Und zwar, indem er zur Debatte stehende Fragestellungen zwar beantwortet, es ihm aber gleichgültig ist, ob die Evidenz, die er in der betreffenden Sache hat, für oder gegen seine Behauptung als eine Antwort auf diese Fragestellung spricht (Fallis und Stokke 2017, S. 295).

Hilfreich ist diese Definition unter anderem deshalb, weil sie verständlich macht, inwiefern Bullshitter in einem anderen Sinn durchaus ein ausgeprägtes Interesse an der Wahrheit ihrer Behauptungen haben können; insbesondere, wenn es sich um Bullshit handelt, dessen Wahrheit ihnen gelegen käme. Dass es sich auch in solchen Fällen um Bullshit handelt, liegt Fallis und Stokke zufolge an der falschen Entsprechungsrichtung (direction of fit) der Behauptungen. Bullshitter sind nicht daran interessiert, ob ihre Evidenz für die Wahrheit oder Falschheit ihrer Behauptungen spricht (mind-to-world direction of fit). Sie wollen höchstens, dass die Dinge so sind, wie sie es behaupten (world-to-mind direction of fit) (Fallis und Stokke 2017, S. 292-297).

Kontexte, in denen Aussagen als Antwort auf eine zur Debatte stehende Fragestellung aufzufassen sind, können wir als Gewährleistungskontexte bezeichnen. In Gewährleistungskontexten gehen Äußerungen automatisch mit der Gewährleistung einher, sich in Fallis und Stokkes Sinn an der Wahrheitsfindung zu beteiligen. ${ }^{4} \mathrm{Ob}$ ein Kontext ein Gewährleistungskontext ist, hängt von den Interessen und Erwartungen der am Diskurs Beteiligten ab und legt fest, ob ein

\footnotetext{
${ }^{3}$ Auch wenn es vermutlich nicht auf jede Art von Diskurs zutrifft, so scheint dies als normative Beschreibung des politischen Diskurses in einer Demokratie hinreichend geeignet. Siehe beispielsweise Habermas (2006), Landemore (2014) und Dryzek et al. (2019).

${ }^{4}$ Wir bedienen uns hier in Kern eines Gedankens, den Carson (2010) unter dem Schlagwort eines warranting context einführt. Carson charakterisiert einen warranting context allerdings als einen Kontext, in dem die Sprecherin automatisch die Wahrheit des Gesagten gewährleistet (2010, 3). Eine solche Gewährleistung kann jedoch streng genommen nie gegeben werden. Bestenfalls kann eine Sprecherin gewährleisten, dass ihre Evidenz nach ihrem Dafürhalten für die Wahrheit des Gesagten spricht. Nur, wenn diese Bedingung nicht erfüllt ist, können wir ihr einen Vorwurf machen. Ob die Behauptung tatsächlich wahr ist, ist nicht entscheidend.
} 
Aussagesatz beispielsweise ein Witz, Teil einer Gutenachtgeschichte oder eine Behauptung über die Welt ist. Allerdings hat nicht jede einzelne Sprecherin jederzeit Autorität über die Art des Kontexts. Es kann durchaus Fälle geben, in denen eine Äußerung in einem Gewährleistungskontext stattfindet, ohne dass sie sich dieser Tatsache bewusst ist oder die Gewährleistung auch tatsächlich geben möchte. Wichtig in unserem Zusammenhang ist, dass Bullshit - wie auch die Lüge - nur in einem Gewährleistungskontext stattfinden kann. Wer bullshittet, muss zunächst einmal eine Behauptung aufstellen. Damit geht die Gewährleistung einher, an der Wahrheitsfindung interessiert zu sein. Allerdings geschieht dies im Falle von Bullshit, ohne dass sich die Sprecherin darum kümmert, ob die Behauptung durch ihre Evidenzen abgedeckt ist.

Nicht jeder akzeptiert die generelle Stoßrichtung der Frankfurt'schen BullshitDefinition. G. A. Cohen (2002) etwa ist der Auffassung, sie stelle die Intentionen der Verbreiter zu sehr in den Vordergrund. Cohen stört sich vorrangig am Bullshit in der Wissenschaft, insbesondere an der französischen Philosophie. Das Problem sei in diesem Fall aber nicht die Gleichgültigkeit der Verfasser gegenüber der Wahrheitsfindung, ${ }^{5}$ sondern die unauflösbare Unklarheit des Gesagten. Diese könne man daran erkennen, dass das Hinzufügen einer Negation keinen Einfluss auf die Plausibilität des Gesagten habe.

Relevant für unsere Zwecke sind an dieser Stelle nicht die Details der Definition Cohens. Wichtig ist, dass man Bullshit auf zwei grundlegend unterschiedliche Weisen charakterisieren kann. Man kann, wie Frankfurt, den Akteur ins Zentrum der Definition stellen und Bullshit unter Rückgriff auf die Haltung charakterisieren, mit der der Bullshitter spricht. Oder aber man kann Bullshit, wie Cohen, anhand von Eigenschaften des Bullshits, also des Produkts selbst, definieren. ${ }^{6} \mathrm{Im}$ ersten Fall können wir von „Frankfurt-Bullshit“ sprechen, im zweiten Fall von

Ein ,warranting context“ ist somit ein Kontext, in dem die Sprecherin garantiert, in Fallis und Stokkes' Sinn zur Wahrheitsfindung beizutragen.

${ }^{5}$ Cohen spricht nicht von „Wahrheitsfindung“, sondern wie Frankfurt von „Wahrheit“. Zugunsten der Einheitlichkeit der Darstellung verwenden wir durchgehend die Terminologie von Fallis und Stokke.

${ }^{6}$ Cohen-Bullshit selbst kann auf drei Arten produziert werden. Er kann erstens unabsichtlich in die Welt kommen, wenn die Sprecherin fälschlicherweise selbst glaubt, dass das, was sie sagt, einen Bedeutungsgehalt hat. Er kann zweitens absichtlich in die Welt kommen, wenn die Sprecherin weiß, dass das, was sie sagt, keinen Bedeutungsgehalt hat, aber ihr Publikum darüber täuschen möchte. Und er kann drittens in die Welt kommen, wenn die Sprecherin ein Frankfurt-Bullshitter ist, der Cohen-Bullshit produziert. Vergleiche dazu auch Cappelen und Dever (2019, S. 61). Relevant für unsere Zwecke ist die Rolle von Frankfurt-Bullshit im aktuellen politischen Zeitgeschehen - unabhängig davon, ob er (zufälligerweise) wahre, falsche oder unauflösbar unklare Aussagen (Cohen-Bullshit) hervorbringt. 
„Cohen-Bullshit“. Wenn wir im Folgenden von „Bullshit“ sprechen, dann verwenden wir den Ausdruck im Frankfurt'schen Sinne - genauer gesagt: in der von Fallis und Stokke ausbuchstabierten Weise.

Ob wir es in einem konkreten Fall mit (Frankfurt'schem) Bullshit zu tun haben, ist nicht immer ganz klar. Frankfurt selbst unterscheidet Bullshit von einem verwandten, aber bei genauerem Hinsehen anderen Phänomen, das er „,bull session“ nennt. Bull sessions sind besondere Gesprächssituationen, in denen die Teilnehmer sich nicht an der Wahrheitsfindung orientieren und diese wahrheitsindifferente Haltung als Modus des Gesprächs anerkannt wird:

While the discussion may be intense and significant, it is in a certain respect not ,for real." $[\ldots]$ What tends to go on in a bull session is that the participants try out various thoughts and attitudes [...] without its being assumed that they are committed to what they say. (Frankfurt 2005, S. 35-37)

Nun ist das besondere an bull sessions, dass im strengen Sinn gar keine Behauptungen aufgestellt werden. Die Teilnehmer gewährleisten mit ihren Äußerungen nicht, dass sie sich an der Wahrheitsfindung beteiligen. Im Gegensatz dazu erfordert Bullshit jedoch einen Gewährleistungskontext: Die geäußerten Sätze müssen Behauptungen sein. Da bull sessions nicht in einem solchen Kontext stattfinden, kann in einer bull sessions (per definitionem) auch nicht gebullshittet werden.

Allerdings sind bull sessions in Reinform überaus selten. In vielen Gesprächssituationen ist nicht ohne weiteres klar, ob sie in einem Gewährleistungskontext stattfinden oder nicht. Witze und klassische Theaterstücke sind recht eindeutige Beispiele: Hier liegt kein Gewährleistungskontext vor. Der Witzbold sagt etwas, um Belustigung zu erzeugen; er legt sich damit nicht auf eine Beteiligung an der Wahrheitsfindung fest. Das Gleiche gilt für die Schauspielerin: Was sie in ihrer Rolle sagt, ist kein Beitrag zur Wahrheitsfindung, sondern die bloße Darstellung eines Sprechakts der Figur. Am anderen Ende der Skala liegen die klaren Fälle von Äußerungen, die in Gewährleistungskontexten stattfinden: Das Experteninterview, die Zeugenaussage und der Schwur sind Beispiele. Die meisten Gesprächssituationen liegen zwischen diesen beiden Extremen. Denken wir etwa an politisches Kabarett, Stammtischdiskussionen oder politische Talkshows. Viele reale Situationen, die bull sessions häufig nur ähneln, gehören plausiblerweise ebenfalls in diese Reihe. Denn dort gehen Gewährleistungskontexte und weniger auf die Wahrheitsfindung orientierte Kontexte in der Regel fließend ineinander über. 


\section{Demonstrativer Bullshit}

Bisher haben wir Bullshit anhand der wahrheitsindifferenten Haltung des Bullshitters herausgegriffen. Insbesondere vor dem Hintergrund der aktuellen politischen Entwicklungen ist aber noch ein weiterer Aspekt der Frankfurt'schen Bullshittheorie wichtig.

Frankfurt ist der Auffassung, Bullshit sei neben der wahrheitsindifferenten Haltung des Bullshitters außerdem dadurch gekennzeichnet, dass Bullshitter versuchen, ihr Publikum über ihre eigene wahrheitsindifferente Haltung zu täuschen. Wie bei einer Lüge sei bei Bullshit also durchaus eine Täuschungsabsicht im Spiel. Im Gegensatz zum Lügner sei der Bullshitter allerdings nicht daran interessiert, über den behaupteten Sachverhalt zu täuschen; vielmehr gehe es ihm darum, das Publikum über seine eigene Haltung zu täuschen, also darüber, dass er nicht an der Wahrheitsfindung interessiert ist. Schließlich, so Frankfurt, können Bullshitter ihre Ziele nur dann erreichen, wenn sie als ernsthafte und nach Wahrheit strebende Akteure wahrgenommen werden.

Doch genau hier geht Frankfurts Definition zu weit, wenn wir am aktuellen politischen Geschehen interessiert sind. Mit seiner Einschränkung auf den angeblich selbstverschleiernden Charakter von Bullshit trifft Frankfurt eine Festlegung, die den Blick darauf verstellt, dass Bullshit im gegenwärtigen politischen Diskurs immer öfter offen zur Schau gestellt wird. Zunehmend zeigt sich dort ein Phänomen, das wir demonstrativen Bullshit nennen wollen: Das Aufstellen von Behauptungen, ohne an der Wahrheitsfindung interessiert zu sein und ohne dabei den Versuch zu unternehmen, die eigene wahrheitsindifferente Haltung zu verbergen.

Bereits die eingangs erwähnten Beispiele von Bullshit sind bei genauerem Hinsehen Fälle demonstrativen Bullshits. Donald Trumps Behauptung, die Sonne habe während seiner Amtseinweihung geschienen, ist ein ebenso klarer Fall demonstrativen Bullshits wie seine Behauptung, die Menschenmenge habe bis zum Washington Monument gereicht. In keinem der beiden Fälle unternimmt Trump den Versuch, seine eigene Gleichgültigkeit gegenüber der Wahrheitsfindung zu verschleiern. Vielmehr stellt er offen zur Schau, dass ihm die Wahrheitsfindung gleichgültig ist.

Trumps Administration hat sich dieses Vorgehen immer wieder zunutze gemacht. So brachte beispielsweise Trumps Beraterin Kellyanne Conway eine Haltung der Wahrheitsindifferenz zum Ausdruck, als sie eine offenkundig falsche Behauptung des Pressesprechers Sean Spicer - auch hier ging es um die Größe der Menschenmenge bei Trumps Amtseinweihung - mit der Aussage rechtfertigte, Spicer habe mit seiner Aussage lediglich ,alternative Fakten“ angeboten (Gajanan 2017). 
Ähnlich argumentierte Newt Gingrich in einem berühmt gewordenen Interview mit der Journalistin Alisyn Camerota. Gefühle, so Gingrich, seien wichtiger als Tatsachen. Als politischer Kandidat habe er die Aufgabe, sich nicht an die Fakten, sondern an die Gefühle der Menschen zu halten (Gingrich 2016).

Auch die Demokratin Alexandra Ocasio-Cortez wurde bereits wegen ihrer Bereitschaft, sich über die Tatsachen hinwegzusetzen, kritisiert. Ocasio-Cortez hatte die Ausgaben des Pentagon falsch dargestellt. In einem Interview mit Anderson Cooper verteidigte sie ihre Falschangaben, indem sie anmerkte: „If people really want to blow up one figure here or one word there, I would argue they are missing the forest for the tree" (Cillizza 2019). Das Problem, so Ocasio-Cortez, sei: ,there's a lot of people more concerned about being precisely, factually, and semantically correct than about being morally right“ (ibid.). „Moralisch richtig“ zu liegen wird hier als höherwertig ausgewiesen als die korrekte Wiedergabe von Tatsachen.

Und auch in Deutschland haben Politiker begonnen, den Wert der Wahrheitsfindung freimütig in Zweifel zu ziehen. So bemerkte der AfD-Pressesprecher Christian Lüth dem Faktenfinder der ARD gegenüber explizit: „Wenn die Message stimmt, ist uns eigentlich egal, woher das Ganze kommt oder wie es erstellt wurde. Dann ist es auch nicht so tragisch, dass es Fake ist" (Becker 2017).

Diese Beispiele legen nahe, unser Bullshitvokabular zu erweitern. Hat der Bullshitter die Absicht, seine Wahrheitsindifferenz vor dem Publikum zu verbergen, können wir von ,selbstverschleierndem Bullshit“ sprechen. Dies ist der Sinn von Bullshit, den Frankfurt stark macht. Demonstrativer Bullshit, um den es uns hier geht, liegt hingegen vor, wenn dem Bullshitter gleichgültig ist, ob das Publikum seine Wahrheitsindifferenz bemerkt oder er sogar die dezidierte Absicht hat, sein Publikum über seine Wahrheitsindifferenz in Kenntnis zu setzen.

Ähnliche Phänomene sind in der Literatur bereits diskutiert worden. Carson (2010, S. 60) zum Beispiel spricht von unverfrorenem Bullshit (,,bald-faced bullshit“). Darunter ist Bullshit zu verstehen, der klar als solcher erkennbar ist und eingesetzt wird, um andere genau damit vor den Kopf zu stoßen. Sein Beispiel ist Bullshit als Mittel der Demütigung:

My transparent open bullshitting is a means of humiliating and angering you. If you protest that my answer is off the topic I will smile and say ,that is my answer". (Carson 2010, S. 60)

Unverfrorener Bullshit ist ein Spezialfall demonstrativen Bullshits. Der unverfrorene Bullshitter ist nicht an der Wahrheitsfindung orientiert, er beabsichtigt, dass sein Gegenüber dies bemerkt, und mit all dem verfolgt er das spezifische Ziel, sein 
Gegenüber zu demütigen oder zu verärgern. Hier erkennt man schon, dass Frankfurt nicht ganz richtig liegt, wenn er darauf hinweist, dass Bullshit selbstverschleiernd sein muss, damit der Bullshitter seine Ziele erreichen kann. Ob das so ist, hängt vielmehr von den spezifischen übergeordneten Zielen des Bullshitters ab.

\section{Demonstrativer Bullshit im politischen Raum: Normen und Deliberation}

Halten wir fest: Demonstrativer Bullshit erfordert keinerlei Täuschungsabsicht weder über den behaupteten Sachverhalt noch über die eigene Gleichgültigkeit gegenüber der Wahrheitsfindung. Ganz im Gegenteil: Der demonstrative Bullshitter spricht, ohne sich an der Wahrheitsfindung zu orientieren, und zeigt seine wahrheitsindifferente Haltung offen. ${ }^{7}$ Darin besteht seine Taktik. Doch was ist sein Ziel?

Auf diese Frage gibt es keine einheitliche Antwort. Im Falle unverfrorenen Bullshits ist das Ziel, das Gegenüber zu verärgern. In der politischen Sphäre lassen sich eine ganze Reihe weiterer Motive für demonstrativen Bullshit festmachen.

Manchmal kann zur Schau gestellte Wahrheitsindifferenz schlichtweg eine Flucht nach vorn in einer ausweglosen Situation sein. Ist man der Falschheit einer Aussage öffentlich überführt, dann bleibt neben dem Eingeständnis des Fehlers nur die unverfrorene Strategie des demonstrativen Bullshittens. Im Krisenmanagement der Trump-Administration erwecken einige Fälle demonstrativen Bullshits den Eindruck, Manöver dieser Art zu sein. Man denke beispielsweise an Trumps Äußerungen im Zusammenhang seines Amtsenthebungsverfahrens. So behauptete er beispielsweise auf Fox \& Friends: „I know Ukrainian people. They're great people. But it's known as being the third most corrupt country in the world" (Qiu 2019). Der Korruptionsvorwurf sollte möglicherweise von seinem versuchten Quidproquo mit dem ukrainischen Präsidenten Wolodymyr Selenskyj ablenken. In jedem Fall ist die Behauptung, dass die Ukraine das drittkorrupteste Land der Welt sei, offenkundig falsch und dies war Trump ebenso offenkundig gleichgültig. Trump scheint hier demonstrativen Bullshit eingesetzt zu haben, um von anderen Ungereimtheiten und Irreführungen abzulenken.

\footnotetext{
${ }^{7}$ Schon die Präzisierung von Frankfurt-Bullshit bei Fallis und Stokke (2017) erfordert keine Täuschungsabsicht. Allerdings wird dort keine besondere Aufmerksamkeit auf demonstrativen Bullshit gelegt. Die Täuschungsabsicht ist lediglich nicht Teil der Definition und die Autoren enthalten sich an dieser Stelle einer Festlegung.
} 
Ein zweites Ziel demonstrativen Bullshits kann in der Demonstration von Macht bestehen. Wer demonstrativ bullshittet, signalisiert, dass er sich in seinem Handeln keinerlei Regeln unterwirft. Der demonstrative Bullshitter sagt, was ihm zupass kommt. Nicht einmal die Wirklichkeit selbst beschränkt ihn in seinen Äußerungen. Dank der Offensichtlichkeit des Bullshittens wird dieser Punkt jedem ersichtlich. Hier sind Aussagen Putins illustrierend, die er im Laufe des Ukraine-Konflikts machte. Im Jahr 2014 behauptete er zunächst, in der Ukraine befänden sich keine russische Soldaten. Etwas später verkündete er dann, es handele sich doch um russische Soldaten, die allerdings nur ihren Urlaub an der Front verbrächten. Nur einen Monat später widersprach er sich erneut, als er betonte, dass es sich natürlich um ,unsere Soldaten“ gehandelt habe. Sie hätten „ganz korrekt gehandelt, sehr entschlossen und professionell“" (Gutschker 2014). Putin scheint hier demonstrativen Bullshit eingesetzt zu haben, um aller Welt und insbesondere seinen Anhängern zu signalisieren, dass ihn niemand für seine Aussagen belangen kann.

Es gibt aber noch mindestens ein weiteres Ziel, dem demonstrativer Bullshit dienen kann und das mit Blick auf das politische Wirken populistischer Akteure von besonderer Bedeutung ist. Demonstrativer Bullshit kann ein wirksames Mittel sein, um die Normen des politischen Diskurses in eine Richtung zu verschieben, die Populisten und Demagogen in die Karten spielt. Demonstrativer Bullshit zerstört nämlich noch direkter unsere, ,zivilisierte Lebensweise und die Lebendigkeit der Institutionen“ (Frankfurt 2002, S. 343), als es selbstverschleiernder Bullshit oder Lügen tun. Wenn politische Akteure öffentlichkeitswirksam und regelmäBig demonstrativ bullshitten, dann widersetzen sie sich damit ganz explizit den Normen der Wahrheitsfindung im gesellschaftlichen Diskurs. Auf diese Weise tragen sie dazu bei, die Grundlage für demokratische Meinungsbildungs- und Entscheidungsprozesse zu zersetzen.

Die RAND Corporation spricht mit Blick auf die USA bereits von einem „Verfall der Wahrheit“ (Kavanagh und Rich 2018). Darunter versteht die Initiative den Anstieg von vier Trends: 1) eine zunehmende Uneinigkeit über Tatsachen und die Interpretation von Tatsachen und Daten, 2) eine zunehmende Verwischung von Meinungen und Tatsachen, 3) einen zunehmenden Einfluss von Meinungen und persönlicher Erfahrung und 4) ein abnehmendes Vertrauen in vormals als vertrauensvoll empfundene Informationsquellen.

Entsprechend dieser Diagnose wird seit einiger Zeit das ,postfaktische Zeitalter" ausgerufen. ${ }^{8}$ Genauer sollte man wohl von einer Prävalenz ,postfaktischer Politik" sprechen. Darunter können wir eine politische Kultur verstehen, in der die

${ }^{8}$ Siehe zum Beispiel Kakutani (2018) oder McIntyre (2018). 
Standards für Wahrheit und Wahrhaftigkeit an Gültigkeit einbüßen. Entsprechend definiert das Oxford Dictionary post-truth in seinem „Word of the Year 2016“Eintrag. Demzufolge, ,betrifft oder bezeichnet [post-truth] Umstände, in denen objektive Tatsachen weniger Einfluss auf die Formung der öffentlichen Meinung haben als Appelle an Gefühle und persönliche Überzeugungen“ (Oxford Dictionaries 2016; unsere Übersetzung). Beinahe dasselbe Phänomen beschreibt Hannah Arendt in Abgrenzung zum Lügen mit dem Begriff defactualization, worunter sie folgendes versteht:

Truth or falsehood - it does not matter which anymore, if your life depends on your acting as though you trusted; truth that can be relied on disappears entirely from public life, and with it the chief stabilizing factor in the ever-changing affairs of men. (Arendt 1972, S. 7)

Arendt weist richtigerweise darauf hin, dass die Orientierung an der Wahrheit ein entscheidender Faktor für das Gelingen des gesellschaftlichen Zusammenlebens darstellt und die demonstrative Gleichgültigkeit gegenüber der Wahrheitsfindung zersetzende Konsequenzen für demokratische Prozesse hat.

Ein Effekt demonstrativen Bullshits besteht darin, dass der politischen Sphäre mehr und mehr der Gewährleistungskontext entzogen wird. Wie David Lewis (1979, S. 347) ausführt, verschieben sich Konversationsnormen systematisch in einer bestimmten Weise, und zwar so, dass sie die tatsächlich auftretenden Sprechhandlungen in einer Konversation als correct play ausweisen. Lewis will hier auf den Punkt hinaus, dass das bloße Vorkommnis einer nach bestehenden Regeln unzulässigen Sprechhandlung einen gewissen Druck auf die Regel selbst ausübt. Wenn viele Teilnehmer einer Debatte unwahrhaftig sprechen und unwahre Aussagen machen, hat das normative Konsequenzen: Unwahrhaftiges Sprechen und falsche Behauptungen werden ein akzeptierter Teil der Debatte. Die Standards dafür, welche Sprechhandlungen zulässig sind, verändern sich. Die Erkenntnisnormen, die sich an Wahrheit und Wahrheitsfindung knüpfen (wie Objektivität, Überprüfbarkeit, Wahrhaftigkeit, Rechtfertigung und dergleichen mehr), erodieren.

Das ist nicht nur ein moralisches Problem. Zwar sind sich die großen deontologischen und konsequentialistischen Moraltheorien einig, dass Lügen und Bullshitten moralisch falsch sind. Entscheidender für unsere Zusammenhänge ist aber, dass die Erosion der genannten Erkenntnisnormen Demokratien erheblichen Schaden zufügen kann. 
Wie Bernard Williams anmerkt, neigen Politiker zu illegitimen Handlungen, die aber ohne eine entsprechende Informiertheit der Bürger nicht aufgedeckt werden können:

Governments are disposed to commit illegitimate actions which they will wish to conceal [...]. It is in citizens' interest that these be checked. They cannot be checked without true information. (Williams 2010, S. 207)

Darüber hinaus beruht die Qualität der öffentlichen Debatte insgesamt wesentlich auf dem Zugang der Bürger zu hinreichend genauen und korrekten Informationen und damit auf der Wahrhaftigkeit derer, die an ihr teilhaben (Fishkin 2009, S. 126; Gutmann \& Thompson, 1996, 81). Versteht man die öffentliche Debatte als entscheidenden Teil des demokratischen Deliberationsprozesses und in dieser Funktion als grundlegend für die Legitimität politischer Entscheidungen, ist Wahrhaftigkeit zudem eine zentrale Bedingung dafür, dass die erfolgten politischen Entscheidungen legitim sind und als solche anerkannt werden sollten (J. Cohen 1997; Habermas 1998, S. 141).

Eine Normenverschiebung, die den Wert der Wahrhaftigkeit in Bedrängnis bringt, ist also eine direkte Bedrohung für das Funktionieren der öffentlichen Debatte und damit für die (deliberative) Demokratie als solche. Man kann davon ausgehen, dass einige populistische Akteure sie aus genau diesem Grund aktiv anstreben. Demonstratives Bullshitting kann ein wirkmächtiges politisches Instrument sein, um demokratische Deliberationsprozesse zu unterminieren. Es lässt sich strategisch einsetzen, um Bullshitting als Form des politischen Diskurses zu etablieren und die Wahrheitsfindungsprozesse der demokratischen Institutionen nach und nach zu unterlaufen.

Möglicherweise ist es aufgrund dieser Mechanismen bei Aussagen Putins inzwischen häufig gar nicht mehr klar, ob sie überhaupt noch in einem Gewährleistungskontext stattfinden. So hat Putin auf einer öffentlich übertragenen Podiumsdiskussion offen „das Geheimnis gelüftet“, dass Russland auch 2020 wieder versuchen werde, US-amerikanischen Wahlen zu manipulieren (Phillips 2019). Hier ist vollkommen unklar, ob diese Aussage ein Fall von Bullshit ist oder ob es sich um einen Witz oder eine andere Art des gewährleistungskontextfreien Sprechens handelt.

Wichtig ist, dass der zersetzende Effekt von Bullshit auch dann besteht, wenn der Bullshitter selbst ganz andere Ziele verfolgt. Was auch immer der Grund für einen Akt demonstrativen Bullshittings im politischen Kontext ist: Wird demonstrativer Bullshit zu einem akzeptierten Beitrag zum politischen Diskurs, geraten die Voraussetzungen für die demokratische Deliberation selbst in Bedrängnis. 


\section{Demonstrativer Bullshit Digital}

Zwar ist demonstrativer Bullshit nicht erst mit dem Internet in die öffentliche Debatte gekommen. Doch hat Bullshit im Allgemeinen - und demonstrativer Bullshit im Besonderen - in der digitalisierten Öffentlichkeit besonders leichtes Spiel. Drei Punkte verdienen in diesem Zusammenhang besondere Beachtung.

Erstens: Der traditionelle Journalismus hat seine Torhüterfunktion weitestgehend eingebüßt. Parteien und andere politische Akteure unterhalten zunehmend eigene Kanäle auf den sozialen Medien und im sogenannten „Dark Net“. Man denke nur an die überdurchschnittlich erfolgreichen Facebook-Accounts vieler AfD-Politiker, die Twitternutzung Donald Trumps oder die zunehmend professionell betriebenen WhatsApp-Gruppen von Unterstützern des brasilianischen Präsidenten Jair Bolsonaro. Auch betreiben politische Akteure weiterhin und zunehmend erfolgreich eigene Medienseiten - wie etwa das US-amerikanische Online-Medium Breitbart oder die russischen Staatsmedien RT und Sputnik. Auf diese Weise können Inhalte direkt in die Newsfeeds der Zielgruppen eingespeist werden. Eine Qualitätskontrolle, bei der die Inhalte auf journalistische Standards wie Wahrhaftigkeit oder Transparenz geprüft werden, findet nicht statt. Einordnungen bleiben aus. Damit existiert kein Fangnetz für (demonstrativen) Bullshit. $^{9}$

Zweitens: In der Aufmerksamkeitsökonomie der digitalen Sphäre finden diejenigen Inhalte am meisten Verbreitung, die am meisten Aufmerksamkeit auf sich ziehen. Demonstrativer Bullshit ist in der Währung der Aufmerksamkeit ein wertvolles Gut. Die offensichtlich nicht an der Wahrheitsfindung orientierten Behauptungen populistischer Akteure finden zuerst im Internet, dann aber auch, nachdem sie meist von den klassischen Medien aufgegriffen wurden, in der öffentlichen Debatte eine überdurchschnittlich hohe Verbreitung. Richtigstellungen und Faktenchecks haben kaum eine Chance, ein vergleichbar großes Publikum zu finden (Shin und Thorson 2017). Die Unterminierung von Wahrheitsnormen ist daher ungleich einfacher als der Schutz der entsprechenden Normen durch klarerweise wahrheitsorientierte Debattenbeiträge.

Drittens: Die Digitalisierung leistet einer tribalistischen Epistemologie Vorschub (Seemann 2017). Darunter kann man die Tendenz von Menschen verstehen, ihre Zugehörigkeit zu einer sozialen Gruppe (tribe) höher zu gewichten als den Gewinn von Erkenntnissen über die Welt. Eine tribalistische Epistemologie wird durch die Digitalisierung besonders begünstigt, weil die Architektur

\footnotetext{
${ }^{9} \mathrm{Ob}$ Bullshit bei einer bestimmten Rezipientengruppe auch verfängt, steht jedoch auf einem anderen Blatt und hängt von verschiedenen weiteren Faktoren ab.
} 
der sozialen Medien Polarisierungsprozesse beschleunigt (Sunstein 2009). Menschen neigen dazu, sich in soziale Umfelder zu selektieren, in denen ihnen möglichst wenig Informationen begegnen, die ihr Überzeugungssystem herausfordern (Mutz 2006, S. 19-56). Auf diese Weise entstehen epistemische Blasen und Echokammern (Nguyen 2018; Quattrociocchi et al. 2016): Soziale Umgebungen, in denen ausschließlich Gleichdenkende miteinander im Austausch stehen. Epistemische Blasen und Echokammern sind die perfekten Umgebungen für Polarisierungsprozesse. Kommunizieren Menschen ausschließlich mit Gleichdenkenden, radikalisieren sie sich in ihren jeweiligen Überzeugungssystemen (Myers und Lamm 1976; Sunstein 2002, 2014; Benkler et al. 2018). In unterschiedlichen Echokammern bilden sich Gruppen heraus, deren Weltbilder stark voneinander abweichen und deren Mitglieder die Weltsicht anderer Gruppen vehement ablehnen. ${ }^{10}$ Ist dieser Punkt erreicht, trumpft Gruppenzugehörigkeit die Orientierung an Normen der Wahrheitssuche.

In diesem Klima kann demonstrativer Bullshit florieren. Behauptungen müssen sich nicht an der Wahrheit orientieren, um in einer tribalistisch-polarisierten Umgebung akzeptiert zu werden. Denn die Disposition, zu den Narrativen der eigenen Gruppe passende Behauptungen zu akzeptieren, ist weit verbreitet (Kahan 2013; Seemann 2017). Demonstrativer Bullshit erfüllt in dieser Umgebung eine klare soziale Funktion, die nichts mit dem Gewinn von Erkenntnissen über die Welt zu tun hat.

Demonstrativer Bullshit kann in einer digitalisierten Gesellschaft also besonders großen Schaden anrichten, weil er dank des Fehlens von Torwächtern besonders viele Menschen erreicht und dem Verfall von Erkenntnisnormen, der durch eine tribalistische Epistemologie bereits in Gang gesetzt ist, mit jeder weiteren Sprechhandlung weiteren Vorschub leistet.

\section{$6 \quad$ Politische Bildung in der Verantwortung}

Vor diesem Hintergrund ergibt sich, dass ein Ziel politischer Bildung darin bestehen sollte, Erkenntnisnormen wie Orientierung an der Wahrheitssuche, Streben nach Wahrhaftigkeit und Einfordern von Rechtfertigung in der öffentlichen Debatte zu stärken. Die Prävalenz demonstrativen Bullshits unterminiert die epistemischen Normen, die für das Funktionieren einer Demokratie, einschließlich

\footnotetext{
${ }^{10}$ In der Forschung wird kontrovers diskutiert, was Echokammern genau auszeichnet und wie schwerwiegend ihre Effekte sind. Siehe zum Beispiel Dubois und Blank (2018) und Bakshy et al. (2015).
} 
des demokratischen Deliberationsprozesses, von zentraler Bedeutung sind. Dieser Entwicklung entgegenzuwirken erfordert zweierlei: eine Vermittlung der Wirkweise demonstrativen Bullshits und eine Stärkung der unter Beschuss stehenden Erkenntnisnormen als solche.

Zum ersten Teil dieser Vorgehensweise leistet der vorliegende Text einen direkten Beitrag. Indem wir aufzeigen, was demonstrativer Bullshit ist und welche Konsequenzen seine Verbreitung hat, stellen wir ein begriffliches Instrumentarium für die Analyse der gesellschaftlichen Situation bereit. Die Herausforderung an die politische Bildung ist aber grundlegender. Neben der Vermittlung erkenntnistheoretischer Zusammenhänge und den Implikationen erkenntnistheoretischer Grundbegrifflichkeiten (wie etwa ,Wahrheit" und ,Wissen“) ist außerdem Aufklärung über die Anreizsysteme der sozialen Medien unabdingbar. Bullshitresistenz, wie Hübl (2018) es nennt, lässt sich nur dann vermitteln, wenn Bürgerinnen und Bürger durchschauen, wie die Anreizsysteme der sozialen Medien, Apps und kommerziellen Informationsanbieter mit den kognitiven Prozessen der eigenen Meinungsbildung im Zusammenhang stehen.

Über die Wirkweise demonstrativen Bullshits aufzuklären, kann jedoch nur der Anfang einer Antwort auf die Probleme sein, die demonstrativer Bullshit in einer digitalisierten Medienumgebung für die Demokratie aufwirft. Peters (2017) weist richtigerweise darauf hin, dass es nicht damit getan ist, Wahrheitsund Erkenntnistheorie zu vermitteln. Vielmehr müssen darüber hinaus Strategien entwickelt werden, die geeignet sind, den erkenntnistheoretischen und ,orwellschen“ Implikationen von ,postfaktischer“ Politik, Wissenschaft und Bildung entgegenzuwirken:

\footnotetext{
We need an operational strategy to combat ,government by lying“ and a global society prepared to accept cognitive dissonance and the subordination of truth to Twittered emotional appeals and irrational personal beliefs. (Peters 2017, S. 3)
}

Wie eine solche operationale Strategie konkret aussehen soll, liegt allerdings nicht auf der Hand. Eine Leitlinie gegen demonstrativen Bullshit sollte aber die direkte Stärkung der unter Beschuss stehenden Erkenntnisnormen selbst sein. Grundsätzlich gilt es, auf ein gesellschaftliches Einvernehmen über den Wert von Wahrheit und Wahrhaftigkeit hinzuwirken. Das beinhaltet unter anderem die Problematisierung von Kommunikationsstrategien, die diese Werte systematisch unterlaufen, und die Stärkung eines selbstständigen, reflektierten und kreativen Umgangs mit diesen Kommunikationsstrategien durch Bürgerinnen und Bürger. Beides erfordert die Förderung der Einsicht, dass Wahrheit ein kostbares Gut ist; dass Bullshit in 
der Politik problematisch, destruktiv und in letzter Konsequenz demokratiegefährdend ist; dass Bürgerinnen und Bürger insbesondere als Nutzerinnen und Nutzer digitaler Medien in der Pflicht stehen, Informationen ausreichend zu prüfen; und dass sie selbiges von anderen Menschen einfordern sollten, indem sie Bullshit und die (stillschweigende) Akzeptanz von Bullshit sozial sanktionieren. Anders gewendet: Es ist eine drängende Aufgabe politischer Bildung, Dispositionen der Bürgerinnen und Bürger zu stärken, sich an der Wahrheitsfindung zu orientieren.

Das kann auf verschiedene Weisen geschehen. Wir wollen drei Punkte herausgreifen, deren weitere theoretische Erörterung, Prüfung und praktische Anwendung sich lohnen dürften: 1) das aktive Vorleben von Erkenntnisnormen durch Vorbilder, 2) die diskursive Auseinandersetzung hinsichtlich des Werts von Erkenntnisnormen und 3) das Etablieren von Praktiken, in denen Erkenntnisnormen Beachtung finden.

Zum ersten Punkt: Normen werden am besten durch Vorbilder, die sie vorleben, gestärkt. Dabei ist essentiell, dass Personen, die die Normen vorleben, in ihrer Vorbildfunktion akzeptiert werden. Diesen Punkt unterstreicht unter anderem Munger (2017). In einer Studie generierte er Social Bots auf Twitter, die männlich aussehen, aber sich in anderen Hinsichten unterscheiden: Sie hatten eine weiße oder schwarze Hautfarbe und wenige oder viele Anhänger. Als Reaktion auf rassistische Äußerungen tweeteten die Bots Ermahnungen, sich nicht rassistisch zu verhalten. Dabei war eine Gruppe von Bots besonders erfolgreich: Weiße Männer, die viele Anhänger hatten.

Diese und weitere Studien zeigen, dass besonderes Augenmerk auf Vorbildern aus dem Lager liegen muss, aus dem demonstrativer Bullshit in die öffentliche Debatte getragen wird. Eine Stärkung der Erkenntnisnormen muss von Personen ausgehen, die von Menschen als Autoritäten anerkannt werden, die Wahrheit und Wahrhaftigkeit nicht bereits als Werte akzeptieren. Damit ist klar, dass sich die politische Bildung dezidiert auch an diejenigen Akteure wenden muss, die eine relevante Vorbildfunktion einzunehmen imstande sind.

Zweitens gibt es eine ganze Reihe einschlägiger Argumente für die grundlegende Rolle, die Erkenntnisnormen für das Funktionieren der deliberativen Demokratie spielen. Politische Bildung kann und soll einen Rahmen bieten, der Bürgerinnen und Bürgern erlaubt, denn Wert darin zu erkennen, politische Diskurse an Erkenntnisnormen wie Objektivität, Überprüfbarkeit, Wahrhaftigkeit oder Rechtfertigung auszurichten. Dazu gehört unter anderem eine Auseinandersetzung mit der Frage, welche Rolle Erkenntnisnormen und Wahrheit als Wert im demokratischen Diskurs spielen. Dass Bürger grundsätzlich zu demokratischer Deliberation in der Lage sind, haben Dryzek et al. (2019) eindrücklich demonstriert. Dass bei der Vermittlung der relevanten Fähigkeiten die Vermittlung von 
Werten zentral ist, die dazu anhalten, sich sowohl im eigenen Denken als auch in der öffentlichen Deliberation praktisch an Erkenntnisnormen zu orientieren, und wie diese Werte didaktisch vermitteln werden können, wurde ebenfalls vielfach gezeigt (etwa von Facione et al. 2000; Kuhn 2010).

Politische Bildung kann und muss hier ihrem eigenen Anspruch gerecht werden und Bürgerinnen und Bürger dazu in die Lage versetzen, Informationen selbst angemessen zu bewerten und durch eigenständige Reflexion und gemeinschaftliche Deliberation begründete politische Standpunkten einzunehmen. Den eigenen Standpunkt aktiv zu hinterfragen, Argumente zu analysieren und kritisch zu diskutieren, sich besonnen und unvoreingenommen mit den Argumenten der Gegenseite auseinanderzusetzen, all dies sind Fähigkeiten, deren Vermittlung sich die politische Bildung zu einem ihrer ersten Ziele setzen sollte. Wie sie sich im negativen Fall gegenseitig schwächen, verstärken sich hier im positiven Fall zwei Faktoren gegenseitig: Die Fähigkeit zum analytischen Denken erleichtert es, die relevanten Erkenntnisnormen zu praktizieren, und das Praktizieren der relevanten Erkenntnisnormen verbessert die Fähigkeit zum analytischen Denken (Bronstein et al. 2019; Pennycook und Rand 2020; Swami et al. 2014).

Drittens fördern die Praktiken der öffentlichen Debatte leider häufig gerade nicht Erkenntnisnormen. Formate wie Facebook- oder Twitter-Threads und WhatsApp-Diskussionsgruppen begünstigen Diskussionen, in denen erkennbar andere Werte im Vordergrund stehen als Wahrheit oder Wahrhaftigkeit. Normale und in der Regel unbedenkliche menschliche kognitive Prozesse wie unsere Tendenz, neue Informationen im Licht unserer bereits bestehenden Überzeugungen zu selektieren und bewerten (Bestätigungsfehler) oder unser Verhalten dem unserer sozialen Gruppe anzupassen (Konformitätsdruck und Tribalismus) sowie aufmerksamkeitsökonomischen Anreize der digitalen und nicht-digitalen Medien machen es in diesen und vielen anderen Fällen fast unmöglich, Wahrheit und Wahrhaftigkeit über andere (Nachrichten-)Werte wie Neuigkeit, Negativität oder Empörungsgehalt zu stellen. In diesen Kontexten kann Bullshit besonders gut florieren.

Hier besteht ganz grundsätzlich die Möglichkeit, Diskursräume zu etablieren, die nach anderen Strukturen funktionieren. So sollte die politische Bildung verstärkt Diskussionsformate wie „Townhall-Meetings“ oder entsprechende Diskussions-Apps fördern. Auch kann sie in stabilen Demokratien, die unabhängige öffentlich-rechtliche Medien unterhalten, den Qualitätsjournalismus dieser von der Marktlogik weniger stark betroffenen Medien unterstützen. Im medial vermittelten Diskurs sollten offen an Erkenntnisnormen orientierte Verhaltensweisen eine Stärkung erfahren. Dazu gehören beispielsweise die Bereitschaft zur ergebnisoffenen Erörterung, das Eingestehen sachlicher Fehler oder die Orientierung an 
Gründen. So gibt es inzwischen auch immer mehr soziale Medien, die sich dezidiert der Aufmerksamkeitsökonomie entziehen oder Nischen etablieren, in denen die Werte der Wahrheit und Wahrhaftigkeit und die dazugehörigen Erkenntnisnormen hochgehalten werden. Dazu zählen beispielsweise (zum Teil kommerzielle) Debatten- und Diskussionsplattformen, aber auch viele Partizipationsplattformen. Diese seitens der politischen Bildung zu stärken, scheint wichtiger denn je. Es gilt nicht nur, mehr solcher Angebote zu fördern, sondern auch, bestehende Formate hinsichtlich ihres Potentials zu evaluieren und zu verbessern, die Werte der Wahrheit und Wahrhaftigkeit sowie die dazugehörigen Erkenntnisnormen zu stärken. ${ }^{11}$

Diese drei Vorschläge skizzieren nur einen kleinen Ausschnitt möglicher Lösungsstrategien für das Problem der Wahrheitsunterminierung im öffentlichen Diskurs. Doch sie zeichnen die Stoßrichtung einer politischen Bildung vor, die der Verbreitung demonstrativen Bullshits - und einer ,postfaktischen“ Politik, in der Wahrheit wertlos wäre - entgegenwirkt. Damit liefert der vorliegende Text einen Anknüpfungspunkt für die weitere theoretische und praktische Auseinandersetzung mit einer individuellen und gesellschaftlichen Bullshitresistenz.

\section{Literatur}

Arendt, H. (1972). Crises of the Republic; lying in politics, civil disobedience on violence, thoughts on politics, and revolution. New York: Harcourt Brace Jovanovich.

Bakshy, E., Messing, S., \& Adamic, L. A. (2015). Political science. Exposure to ideologically diverse news and opinion on Facebook. Science, 348(6239), 1130-1132. https://doi.org/ $10.1126 /$ science.aaa1160

Becker, K. (2017). AfD teilt falsches Antifa-Foto: Fake? Egal! „Es geht um die Message“. Tagesschau. https://web.archive.org/web/20170720182846/http://faktenfinder.tagess chau.de/inland/falsches-antifa-foto-101.html.

Benkler, Y., Faris, R., \& Roberts, H. (2018). Network propaganda: manipulation, disinformation, and radicalization in American politics. New York: Oxford University Press.

Bronstein, M. V., Pennycook, G., Bear, A., Rand, D. G., \& Cannon, T. D. (2019). Belief in fake news is associated with delusionality, dogmatism, religious fundamentalism, and reduced analytic thinking. Journal of Applied Research in Memory and Cognition, 8(1), 108-117. https://doi.org/10.1016/j.jarmac.2018.09.005.

Cappelen, H., \& Dever, J. (2019). Bad language. Oxford: Oxford University Press.

\footnotetext{
${ }^{11}$ Dabei sind auch die ersten beiden Vorschläge wieder einschlägig. So bräuchte es entsprechende Onlineangebote, die gerade auch für Menschen attraktiv sind, die zunächst einmal wenig mit den Normen der Wahrheit und Wahrhaftigkeit anfangen können. Und sie müssten so funktionieren, dass diese Normen tatsächlich an die Nutzer vermittelt werden.
} 
Carson, T. L. (2006). The definition of lying. Noûs, 40(2), 284-306.

Carson, T. L. (2010). Lying and deception. Theory and practice. Oxford: Oxford Scholarship.

Carson, T. L. (2016). Frankfurt and Cohen on bullshit, bullshitting, deception, lying, and concern with the truth of what one says. Pragmatics \& Cognition, 23(1), 53-67.

Cillizza, Chris. (2019, 10. Jan.). Alexandra Ocasio-Cortez' very slippery slope on facts. CNN. https://edition.cnn.com/2019/01/07/politics/alexandria-ocasio-cortez-facts/index.html.

Cohen, G. A. (2002). Deeper into bullshit. In S. Buss \& L. Overton (Hrsg.), The contours of agency: Essays on themes from Harry Frankfurt (S. 321-339). Cambridge, MA: MIT Press.

Cohen, J. (1997). Deliberation and democratic legitimacy. In J. Bohman \& W. Rehg (Hrsg.), Deliberative democracy: Essays on reason and politics (S. 67-92). Cambridge, MA: MIT Press.

Dryzek, J. S., Bächtiger, A., Chambers, S., Cohen, J., Druckman, J. N., Felicetti, A., \& Warren, M. E. (2019). The crisis of democracy and the science of deliberation. Science, 363(6432), 1144-1146. https://doi.org/10.1126/science.aaw2694.

Dubois, E., \& Blank, G. (2018). The echo chamber is overstated: the moderating effect of political interest and diverse media. Information, Communication \& Society, 21(5), 729-745.

Facione, P., Facione, N., \& Giancarlo, C. (2000). The disposition toward critical thinking: its character, measurement, and relationship to critical thinking skill. Informal Logic, 20(1).

Fallis, D. (2009). What is lying? Journal of Philosophy, 106(1), 29-56.

Fallis, D., \& Stokke, A. (2017). Bullshitting, lying, and indifference toward truth. Ergo, 4(10), 277-309.

Fishkin, J. S. (2009). When the people speak: Deliberative democracy and public consultation. Oxford: Oxford University Press.

Frankfurt, H. G. (1986). On bullshit. . Raritan Quarterly Review, 6(2), 81-100.

Frankfurt, H. G. (2002). Reply to G. A. Cohen. In S. Buss \& L. Overton (Hrsg.), The contours of agency: Essays on themes from Harry Frankfurt (S. 340-344). Cambridge: MIT Press.

Frankfurt, H. G. (2005). On bullshit. Princeton: Princeton University Press.

Frankfurt, H. G. (2016, 12. Mai). Donald Trump is BS, says expert in BS. Time. https://time. com/4321036/donald-trump-bs.

Gajanan, M. (2017, 22. Jan). Kellyanne Conway defends White House's falsehoods as ,alternative facts“. Time. https://time.com/4642689/kellyanne-conway-sean-spicer-donaldtrump-alternative-facts.

Gingrich, N. (2016, 22. Juli). Was Donald Trump's speech too dark? Newt Gingrich clarifies comments on sharia law. CNN. https://transcripts.cnn.com/TRANSCRIPTS/1607/22/ nday.06.html.

Gutschker, T. (2014). Der Kreml und die Wahrheit: Putins Lügen. Frankfurter Allgemeine Zeitung. https://www.faz.net/aktuell/politik/ausland/derkreml-und-die-wahrheitputins-luegen-13002460.html.

Habermas, J. (1998). Faktizität und Geltung: Beiträge zur Diskurstheorie des Rechts und des demokratischen Rechtsstaats. Frankfurt a. M.: Suhrkamp.

Habermas, J. (2006). Political communication in media society: does democracy still enjoy an epistemic dimension? the impact of normative theory on empirical research. Communication Theory, 16(4), 411-426. 
Isenberg, A. (1973). Deontology and the ethics of lying. In W. Callaghan, L. Cauman, C. G. Hempel, S. Morgenbesser, M. Mothersill, E. Nagel, \& T. Norman (Hrsg.), Aesthetics and the theory of criticism. Selected essays of Arnold Isenberg (S. 245-264). Chicago: University of Chicago Press.

Kahan, D. H. (2013). Ideology, motivated reasoning, and cognitive reflection. Judgment and Decision Making, 8(4), 407-424.

Kakutani, M. (2018). The death of truth. London: William Collins.

Kavanagh, J., \& Rich, M. D. (2018). Truth decay: an initial exploration of the diminishing role of facts and analysis in American public life. Santa Monica: RAND Corporation.

Kimbrough, S. (2006). On letting it slide. In G. L. Hardcastle \& G. A. Reisch (Hrsg.), Bullshit and philosophy (S. 3-18). Chicago: Open Court.

Kuhn, D. (2010). Teaching and learning science as argument. Science Education, 94, 810-824. https://doi.org/10.1002/sce.20395.

Landemore, H. (2014). Truth and democracy. Contemporary Political Theory, 13(2), 7-11.

McIntyre, L. C. (2018). Post-truth. Cambridge: MIT Press.

Munger, K. (2017). Tweetment effects on the tweeted: experimentally reducing racist harassment. Political Behavior, 39(3), 629-649.

Mutz, D. C. (2006). Hearing the other side: deliberative versus participatory democracy. Cambridge, MA: Cambridge University Press.

Nguyen, C. T. (2018). Echo chambers and epistemic bubbles. Episteme, 1,. https://doi.org/10. 1017/epi.2018.32.

Myers, D. G., \& Lamm, H. (1976). The group polarization phenomenon. Psychological Bulletin, 83(4), 602-627. https://doi.org/10.1037/0033-2909.83.4.602.

Oxford Dictionaries (2016). Word of the Year 2016. https://languages.oup.com/word-of-theyear/2016/.

Pennycook, G., \& Rand, D. G. (2020). Who falls for fake news? The roles of bullshit receptivity, overclaiming, familiarity, and analytic thinking. Journal of Personality, 88(2), 185-200.

Phillips, J. (2019, 2. Okt.). „Don't tell anyone“: Putin jokes about Russia interfering in 2020 election. The Epoch Times. https://www.theepochtimes.com/dont-tell-anyone-putinjokes-about-russia-interfering-in-2020-election_3103983.html.

Quattrociocchi, W., Scala, A., \& Sunstein, C. R. (2016). Echo Chambers on Facebook. http:// dx.doi.org/https://doi.org/10.2139/ssrn.2795110.

Saul, J. M. (2012). Lying, misleading, and what is said. An exploration in philosophy of language and in ethics. Oxford: Oxford University Press.

Seemann, Michael. (2017, 29. September). Digitaler Tribalismus und Fake News [Blog Post]. https://www.ctrl-verlust.net/digitaler-tribalismus-und-fake-news.

Stalnaker, R. (2002). Common ground. Linguistics and Philosophy, 25(5/6), 701-721.

Sunstein, C. R. (2002). The law of group polarization. Journal of Political Philosophy, 10(2), 175-195.

Sunstein, C. R. (2009). Republic 2.0. Princeton, NJ: Princeton University Press.

Sunstein, C. R. (2014). On rumors. how falsehoods spread, why we believe them, and what can be done. Princeton, NJ: Princeton University Press.

Swami, V., Voracek, M., Stieger, S., Tran, U. S., \& Furnham, A. (2014). Analytic thinking reduces belief in conspiracy theories. Cognition, 133(3), 572-585. https://doi.org/10.1016/ j.cognition.2014.08.006. 
Peters, M. A. (2017). Education in a post-truth world. Educational Philosophy and Theory, 49(6), 563-566.

Shin, J., \& Thorson, K. (2017). Partisan selective sharing: the biased diffusion of fact-checking messages on social media. Journal of Communication, 67(2), 233-255.

Williams, B. (2010). Truth and truthfulness. An essay in genealogy. Princeton: Princeton University Press.

Wreen, M. (2013). A P.S. on B.S. Some remarks on humbug and bullshit. Metaphilosophy, 44(1-2), 105-115.

Qiu, L. (2019). Trump's long list of inaccurate statements on „Fox \& Friends“. New York Times. https://www.nytimes.com/2019/11/22/us/politics/trump-fox-and-fri ends-fact-check.html.

Lewis, D. K. (1979). Scorekeeping in a Language Game. Journal of Philosophical Logic 8, 339-359.

Open Access Dieses Kapitel wird unter der Creative Commons Namensnennung 4.0 International Lizenz (http://creativecommons.org/licenses/by/4.0/deed.de) veröffentlicht, welche die Nutzung, Vervielfältigung, Bearbeitung, Verbreitung und Wiedergabe in jeglichem Medium und Format erlaubt, sofern Sie den/die ursprünglichen Autor(en) und die Quelle ordnungsgemäß nennen, einen Link zur Creative Commons Lizenz beifügen und angeben, ob Änderungen vorgenommen wurden.

Die in diesem Kapitel enthaltenen Bilder und sonstiges Drittmaterial unterliegen ebenfalls der genannten Creative Commons Lizenz, sofern sich aus der Abbildungslegende nichts anderes ergibt. Sofern das betreffende Material nicht unter der genannten Creative Commons Lizenz steht und die betreffende Handlung nicht nach gesetzlichen Vorschriften erlaubt ist, ist für die oben aufgeführten Weiterverwendungen des Materials die Einwilligung des jeweiligen Rechteinhabers einzuholen. 